\title{
«Wer nicht handelt, wird behandelt»
}

\author{
Als ehemaliger Vorsitzender der deutschen «Ärztegewerkschaft» Marburger Bund und \\ aktueller Präsident der Bundesärztekammer ist Frank Ulrich Montgomery vielen \\ Schweizer Ärztinnen und Ärzten ein Begriff. Im Zug eines Gastauftritts an der Ärzte- \\ kammer vom vergangenen Oktober in Biel, bei dem er für seine Rede von den Delegier- \\ ten grossen Applaus erhielt, gewährte er der SÄZ das folgende Interview. Im Zentrum \\ standen Fragen zu Arztberuf und Gesundheitswesen, die beide Länder betreffen.
}

Interview: Bruno Kesseli

\footnotetext{
* In einem Grundsatzurteil vom 3. Oktober 2000 («Simap-Entscheidung») hat der Europäische Gerichtshof im Zusammenhang mit dem Bereitschaftsdienst spanischer Ärzte entschieden, dass die Bereitschaftszeit in Form der Anwesenheitsbereitschaft Arbeitszeit im Sinne des europäischen Arbeitszeitrechts ist. Danach ist die Bereitschaftszeit bei der Berechnung der zulässigen Höchstarbeitszeit voll zu berücksichtigen. Die Bereitschaftszeit ist somit keine Ruhezeit, selbst wenn die Arbeitsleistung des Arztes im Bereitschaftsdienst nicht in Anspruch genommen wird Dagegen sind Zeiten, in denen lediglich Rufbereitschaft geleistet wird, nicht als Arbeitszeit in dem genannten Sinne anzusehen.
}

Wenn Sie an das Jahr 2006 zurückdenken, welche Erinnerungen kommen da bei Ihnen auf?

Frank Ulrich Montgomery: Sie sprechen den Ärztestreik an?

Genau.

Nun, dahinter stand ein längerer Prozess, der von 2000 bis 2006 dauerte. Man soll nicht glauben, dass man eine solche grosse Streik- und Veränderungsaktion ohne sehr viel Vorarbeit zustande bringt. Den Ausgangspunkt bildete ein Urteil des Europäischen Gerichtshofs im Jahr 2000* zur Arbeitszeit, das uns sehr in die Hände spielte. In der Folge haben wir

\section{«Die allgemeine Stimmung war:}

¿Da muss man jetzt was tun, damit es diesen Leuten besser geht.»»

konsequent Aktionen aufgebaut, insbesondere der jungen Ärztinnen und Ärzte, aber auch der Oberärzte in den Spitälern, um darauf hinzuweisen, dass Ärzte zu viel arbeiten müssen und zu wenig verdienen.

\section{Mit eindrücklichem Erfolg.}

Irgendwann waren eben nicht mehr nur die Ärzte auf Zinne, sondern war auch die Bevölkerung davon überzeugt, dass gerade die jungen Ärzte zu viel arbeiten für zu wenig gesellschaftliche Anerkennung. Als wir das geschafft hatten, wurden auch unsere Aktionen wie Streiks oder Demonstrationen positiv in der Bevölkerung aufgenommen. Entscheidend war nicht der ökonomische Schaden, der den Krankenhäusern aus diesen Aktionen und der reduzierten Patientenversorgung entstand. Vielmehr kippte die

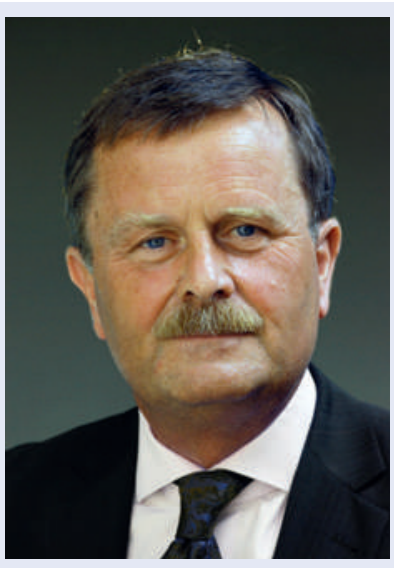

Frank Ulrich Montgomery ist seit 2011 Präsident der Bundesärztekammer.

öffentliche Meinung auf unsere Seite, und die allgemeine Stimmung war: «Da muss man jetzt was tun, damit es diesen Leuten besser geht.»

Was haben Sie denn Positives für die Ärzteschaft erreicht?

In den Jahren 2006 und 2007, also am Ende dieses langen Prozesses, standen Tarifverträge. Unter dem Strich haben diese den Assistenzärzten ungefähr 20 Prozent mehr Einkommen, vor allem aber eine erhebliche Reduktion der Arbeitszeit gebracht. Die Verhältnisse sind zwar immer noch nicht perfekt, aber sehr viel besser als vor diesem Prozess. Das kann durchaus als Erfolg gewertet werden, und ich bezweifle, dass das heute aus dem Stand wieder möglich wäre.

\section{Weshalb nicht?}

Weil das Potential der Unzufriedenheit nicht so gross ist wie damals. Damals kamen einfach drei Dinge zusammen: Es gab zum einen eine öffentliche 
Bewegung, ausgelöst durch das genannte Gerichtsurteil. Zum anderen ein Unruhepotential bei den jungen Ärzten. Drittens waren dann zufälligerweise gerade ich und der Marburger Bund ${ }^{* *} \mathrm{da}$, um die Anliegen dieser jungen Ärzte zu vertreten.

\section{«Ich war schon in der Schule einer,} der «die Schnauze aufgemacht> hat.»

\section{Waren diese Erfolge aus heutiger Sicht nachhaltig?}

Die Tarifverträge werden bis heute fortgeschrieben. Und in den letzten Jahren hat dann noch eine vierte Komponente mit hineingespielt: Die Veränderungen im Bereich der Arbeitszeit, aber auch die Generations- und Genderveränderungen haben dazu geführt, dass die ärztliche Arbeitskraft knapper geworden ist. Diese Faktoren haben dazu beigetragen, das Erreichte nachhaltig zu gestalten. Die Krankenhäuser führen heute Tarifverhandlungen mit uns auf einer ganz normalen Arbeitgeber-Arbeitnehmer-Basis, und sie haben durchaus im Hinterkopf, dass wir auch wieder einmal streiken könnten.

Wie kamen Sie persönlich eigentlich dazu, sich standespolitisch zu engagieren?

Ich war schon in der Schule einer, der «die Schnauze aufgemacht» hat. Obwohl ich aus einem relativ grossbürgerlichen Haus stamme, habe ich mich über Ungerechtigkeiten wahnsinnig aufgeregt. Wenn

\section{«Ich hatte Chefs, die Verständnis für meine berufs-}

\section{politischen Tätigkeiten hatten und mich darin bestärkt} und unterstützt haben.»

\footnotetext{
** Der Marburger Bund ist die gewerkschaftliche, gesundheits- und berufspolitische Interessenvertretung aller angestellten und beamteten Ärztinnen und Ärzte in Deutschland.
}

Menschen ungerecht behandelt worden sind, habe ich immer etwas dagegen gesagt. So wird man eben Klassensprecher, Schulsprecher - und plötzlich war ich Vorsitzender des Marburger Bundes, später Präsident der Ärztekammer Hamburg und jetzt Präsident der Bundesärztekammer. Natürlich gehört dazu ein Schuss Narzissmus, ein Schuss Ehrgeiz - vor allem aber braucht es die Bereitschaft, viel zu arbeiten.

Neben Ihren berufspolitischen Aktivitäten arbeiten Sie weiterhin auch als Arzt.

Es gibt ja das Vorurteil, dass diejenigen, die Standespolitik machen, nicht mehr in der eigentlichen Medizin tätig sind. Ich habe bis heute immer beides gemacht, wobei ich natürlich das Glück hatte und habe, in einer sehr grossen Abteilung zu arbeiten, in der das möglich ist. Ich hatte Chefs, die Verständnis

\section{Frank Ulrich Montgomery}

1952 als Sohn einer Hausärztin und eines britischen Offiziers in Hamburg geboren, wuchs Frank Ulrich Montgomery in der Hansestadt an der Elbe auf und absolvierte dort sowie in Sydney, Australien, sein Medizinstudium. Nach der Approbation und Promotion 1979 folgte die Weiterbildung zum Facharzt für Radiologie, die er 1986 abschloss. Trotz seines grossen standespolitischen Pensums ist Montgomery bis heute auch als Arzt tätig, aktuell in Teilzeitanstellung als Oberarzt am Universitätsklinikum HamburgEppendorf.

Seit 1983 ist er Vorsitzender der «Ärztegewerkschaft» Marburger Bund** in Hamburg. Von 1989 bis 2007 war er 1. Vorsitzender des Bundesverbands Marburger Bund, zu dessen Ehrenvorsitzendem er 2007 ernannt wurde. Von 1994 bis 2002 war er Präsident der Ärztekammer Hamburg, die er seit 2006 wieder präsidiert. Bereits 1987 war er in den Vorstand der Bundesärztekammer gewählt worden, wo er zunächst bis 2002 aktiv war und dem er seit 2006 erneut angehört. Von 2007 bis 2011 war er Vizepräsident der Bundesärztekammer, der er seit 2011 als Präsident vorsteht. Frank Ulrich Montgomery ist verheiratet mit einer niedergelassenen Allgemeinärztin. Das Ehepaar hat zwei Kinder und lebt in Hamburg.

für meine berufspolitischen Tätigkeiten hatten und mich darin bestärkt und unterstützt haben.

Obwohl Beachtliches erreicht wurde, ist für die Ärzte in Deutschland - wie in der Schweiz - bei weitem nicht alles eitel Sonnenschein. Wo sehen Sie aus der Perspektive der Ärzteschaft die drängendsten Probleme im deutschen Gesundheitswesen?

Eine grosse Herausforderung ist die demographische Entwicklung in der Bevölkerung. Wir werden immer älter, was eine Intensivierung der pflegerischen Betreuung nach sich zieht. Darauf sind unsere Systeme noch nicht eingestellt. Wenn in einem Umlageverfahren die Arbeitenden die Rentner unterstützen, dann funktioniert das System heute, weil drei Arbeitende einen Rentner finanzieren. Wenn aber unsere Kinder aufgrund der demographischen Entwicklung irgendwann 60-70 Prozent ihres Bruttoeinkommens abgeben müssen für die Alters- und Krankenversorgung ihrer Eltern, werden sie dieses System nicht mehr mittragen.

\section{Was schlagen Sie vor?}

Wir müssen heute Vorsorge tragen - und Vorsorge heisst für mich Kapitalbildung - damit jeder seine eigene Krankenversicherung selber bezahlen kann und nicht darauf angewiesen ist, dass zukünftige Generationen ihm dies abnehmen. Die Negativspirale, 


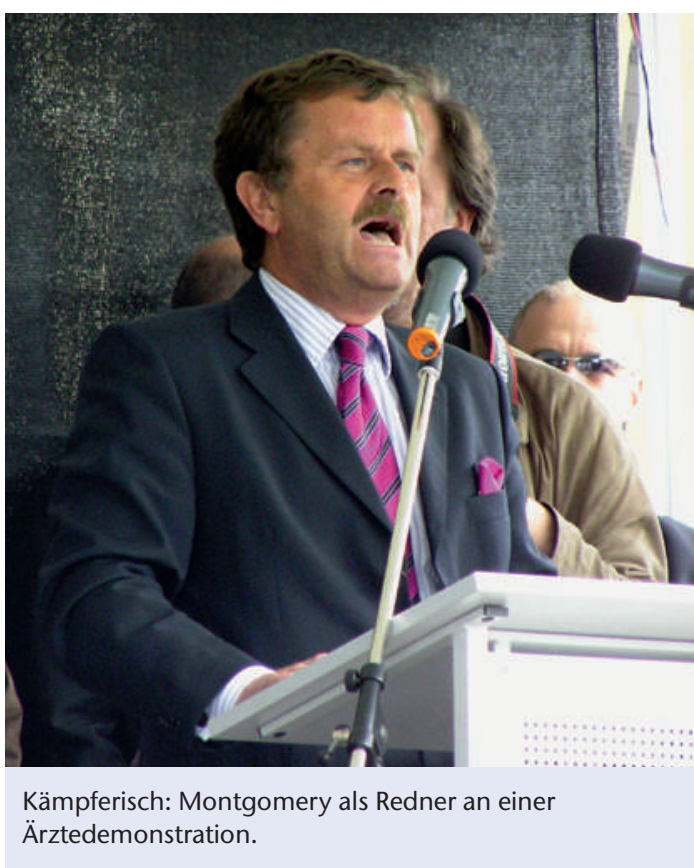

in der wir uns zurzeit bewegen, kann nur über andere Finanzierungssysteme unterbrochen werden. Konkret, indem man ein prämienfinanziertes System einführt und den Solidarausgleich über Steuern macht.

In der Schweiz sind die Kosten im Gesundheitswesen ein Dauerbrenner.

Das ist in Deutschland nicht anders, wobei ich als Problemfeld in diesem Zusammenhang die Implementierung des medizinischen Fortschritts nennen möchte. Man kann für Deutschland berechnen, bewältigen und finanzieren. Dazu brauchen wir mehr Leute, bessere Arbeitsplätze und mit Sicherheit auch mehr Geld.

Ökonomische Fragestellungen sind in der Medizin in Deutschland wie in der Schweiz im Berufsalltag quasi omnipräsent. Die Ärzteschaft empfindet sich nicht mehr als Herr im eigenen Haus, was als ein Aspekt der sogenannten Deprofessionalisierung gesehen werden kann.

Beide Seiten sind hier gefordert. Die Ökonomen müssen die Mediziner besser verstehen, die Mediziner müssen die Ökonomen besser verstehen. Die Verteufelung der Ökonomie halte ich für Unsinn. Der Ökonom macht seinen Job, so wie wir unseren Beruf ausüben. Zunächst sollte sich jede Seite bemühen, die Sprache des anderen zu verstehen. Zweitens sollte man sich mit den unterschiedlichen Logikgebäuden befassen.

\section{Was meinen Sie damit?}

Dem Ökonomen ist in der Regel das Individuum, der einzelne Patient, völlig egal. Ihn interessiert, wie man mit dem geringsten Mittelaufwand zum besten Ergebnis kommt. Dazu vergleicht er zum Beispiel zwei Cholezystektomie-Verfahren bei mehreren hundert Patienten. Ich als Arzt habe aber die Verpflichtung, meinen Patienten, der sehr subjektive Ansichten darüber hat, was er als Ergebnis haben will, individuell zu beraten. Da nützen MittelwertAussagen nicht sehr viel. Diese Spannung zwischen ökonomischer und medizinischer Sichtweise muss man sich immer wieder vor Augen führen - beide Perspektiven haben ihre Berechtigung.

\section{«Wir müssen uns darauf einstellen, dass mehr Ärzte weniger arbeiten, aber natürlich gleich viel verdienen wollen.»}

dass die Kostensteigerung im Gesundheitswesen ausschliesslich auf die Innovationsschübe in der Medizin zurückzuführen ist. Da wir in der Medizin immer mehr können, werden die Kosten auch weiter zunehmen - wir können die Medizin ja nicht auf dem Stand von 2013 einfrieren. Es wird laufend neue Entwicklungen geben, die wir für die Patienten umsetzen müssen und wollen. Diese Entwicklungen wollen finanziert sein.

Auch die Ärztedemographie dürfte in beiden Ländern ein Thema sein.

Auf jeden Fall. Die Medizin wird ein zunehmend weiblicher Beruf. Frauen haben andere Arbeitsrhythmen als Männer. Wir müssen uns darauf einstellen, dass mehr Ärzte weniger arbeiten, aber natürlich gleich viel verdienen wollen. Unter diesen Vorzeichen müssen wir dieselbe oder noch mehr Arbeit
Ist unter diesen Voraussetzungen eine fruchtbare Zusammenarbeit überhaupt möglich?

Der Grundkonflikt zwischen den beiden Disziplinen wird immer bestehen. Auch der Arzt darf ökonomische Prinzipien nicht vernachlässigen, wenn es um die Rahmenbedingungen seiner Arbeit geht. Wenn die Möglichkeit besteht, mit Hilfe der Ökonomie Prozesse zu verbessern, sollten sich die Ärzte einbringen, anstatt alles den Ökonomen zu überlassen und hinterher darüber zu meckern, dass sie nicht beteiligt waren. Wer lange verheiratet ist, weiss im Übrigen, dass man auch mit Menschen, die man sehr gern hat, Konflikte austragen muss. Man löst die Konflikte, aber man geht nicht gleich zum Scheidungsrichter.

Ein weiterer Bereich, in dem sich deutsche und schweizerische Erfahrungen vergleichen lassen, sind die DRG. In Deutschland ist das Fallpauschalen-System in der sta- 


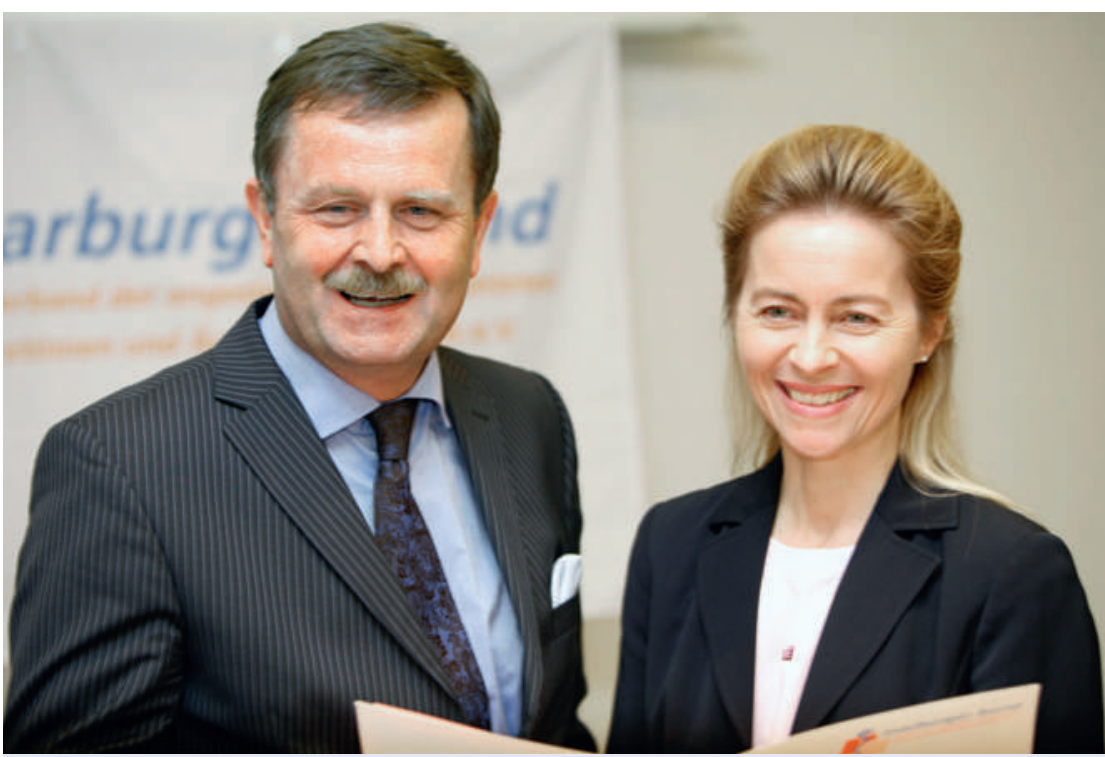

Politisch aktiv: Montgomery mit der deutschen Bundesministerin Ursula von der Leyen.

tionären Medizin schon länger Realität. Würden Sie ein anderes System vorziehen?

Sowohl Systeme, die Einzelleistungen vergüten, als auch Systeme mit Tagespauschalen setzen fragwürdige Anreize. Deswegen gibt es aus meiner Sicht keine Alternative zu einem Fallpauschalen-System. Ob ein Fallpauschalen-System klug und gut gemacht ist, ist eine Frage der Ausgestaltung. Ob es 600 Fallpauschalen braucht oder 4000 , ob man Zuschläge braucht für Notfälle, für Kinder, für besonders alte Menschen, für Unikliniken usw. - das alles sind Fragen der Ausgestal-
$\mathrm{Zu}$ den Themen, die die Schweizer Ärzteschaft bewegen und die teilweise auch polarisieren, zählt die «Einheitskasse», über deren Einführung das Schweizer Volk in absehbarer Zeit abstimmen wird. In Deutschland läuft dieselbe Diskussion unter dem Stichwort «Bürgerversicherung». Was ist Ihre Meinung dazu?

Eine Versicherung aller Bürger haben wir ja in Deutschland längst, und mit «Bürgerversicherung» ist in der Tat eine Einheitskasse gemeint. Würde Sie eingeführt, entfiele der aktuelle Wettbewerb zwischen privaten Krankenversicherungen und der gesetzlichen Krankenversicherung. Dieser Wettbewerb führt aber dazu, dass die gesetzliche Krankenversicherung sehr viel patientenorientierter ist, neue Verfahren sehr viel früher einführt, als dies ohne Wettbewerb der Fall wäre.

\section{Wie ist das zu verstehen?}

Wenn privat Versicherte medizinisch sinnvolle Leistungen erhalten, gesetzlich Versicherte dagegen nicht, so erzeugt dies einen Druck auf die gesetzliche Krankenversicherung. Dieser Druck führt dazu, dass sinnvolle Neuerungen schneller generell zugelassen werden und damit der Allgemeinheit zugute kommen. Wenn man diesen Wettbewerb ausschaltet, ist der Innovationsdruck aus unserem Versicherungssystem raus. Wir haben es dann mit einem monolithischen Block von Kassenvertretern zu tun. Darunter werden nicht nur die Ärzte, sondern vor allem die Patienten leiden. Denn für diese Funktionäre besteht dann kein Druck, neue, sinnvolle Leistungen rasch in den Leistungskatalog aufzunehmen.

\section{«Nicht darüber jammern, dass wir von der Politik schlecht behandelt werden, sondern selbst in die Politik gehen!»}

tung. Darüber kann man reden, das muss man regeln. Es ist aber falsch, das DGR-System an sich als schlecht zu bezeichnen, wenn es schlecht ausgestaltet ist.

\section{Wie läuft die Anpassung des Systems in Deutschland?} Sind Sie damit zufrieden?

Die Anpassung funktioniert, auch wenn beide Seiten, das heisst Ärzteschaft und Versicherer, nicht vollständig glücklich sind. Wenn bei einer Kompromissfindung aber beide Seiten nicht ganz zufrieden sind, ist der Kompromiss wahrscheinlich nicht so schlecht. Allerdings gibt es in Deutschland eine wichtige Differenz zur Schweiz. Via Fallpauschalen werden nur die Betriebskosten der Spitäler gedeckt, nicht aber die Investitionskosten. Es handelt sich also nicht um ein monistisches System, was zu einer Reihe spezifischer Probleme führt, die in der Schweiz in dieser Art nicht auftreten.
Wie wichtig ist es aus Ihrer Sicht für die Ärzteschaft, sich politisch zu engagieren? Sowohl in Deutschland als auch in der Schweiz sind Ärztinnen und Ärzte im nationalen Parlament ziemlich dünn gesät.

Ich sage meinen Kolleginnen und Kollegen immer, dass wir uns viel stärker politisieren müssen. Tatsächlich bin ich selbst auch viel politischer als meine Vorgänger. Wir müssen einerseits in die Parteien hineinwirken, aber auch nach aussen klar und deutlich sagen, was wir wollen. Und auf unseren Ärztetagungen sollten wir nicht nur über die Verwaltung des Mangels diskutieren, sondern auch darüber, wie wir verhindern können, dass der Mangel überhaupt entsteht. Also nicht darüber jammern, dass wir von der Politik schlecht behandelt werden, sondern selbst in die Politik gehen! Wer nicht handelt, wird behandelt! 